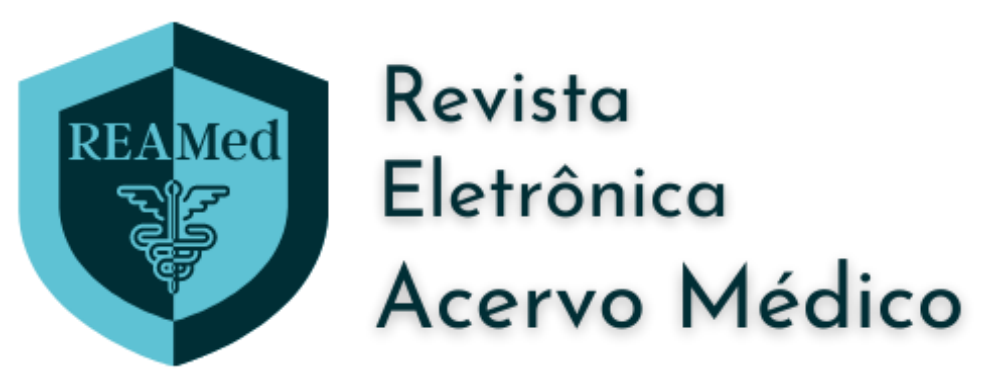

\title{
Síndrome de Usher tipo 2 surdez congênita e visão subnormal no adulto: relato de caso
}

Usher syndrome type 2 congenital deafness and subnormal vision in the adult: case report

Síndrome de Usher tipo 2 sordera congénita y visión subnormal en el adulto: informe de caso

Vinicio Tadeu da Silva Coelho ${ }^{*}$, Marlon Nielsen de Araújo, Emílio Castellar Macedo Foureaux.

\section{RESUMO}

Objetivo: Descrever o diagnóstico tardio de um caso de Síndrome de Usher (SU), analisar as manifestações clínicas e classificar a doença utilizando os achados oftalmológicos além de compará-los à literatura atual. Detalhamento do caso: Mulher de 32 anos, veio à consulta queixando-se de baixa Acuidade Visual (AV) com piora da visão noturna. Apresentava lupus, hipertensão arterial sistêmica dislipidemia, hipoacusia neurossensorial em uso de aparelho, cirurgias oculares prévias, faco+lio em 2009 e 2016. Ao exame AV 20/50 no Olho Direito (OD) e 20/60 no Olho Esquerdo (OE) com lentes corretivas, tonometria $10 \mathrm{mmhg}$ OD e 13 mmh OE, biomicroscopia sem alteração em Ambos os Olhos (AO). À campimetria apresentou, perda de Campo Visual (CV) periférico em AO. À fundoscopia demonstrou discos ópticos pálidos, estreitamento vascular e espículas ósseas em AO. Considerações finais: A SU se caracteriza pela associação de Retinose Pigmentar (RP) e surdez congênita, parcial ou total. É uma doença autossômica, herd ada recessivamente. Os achados clínicos juntamente com os exames complementares apoiaram para o diagnóstico de RP e SU subtipo 2. O diagnóstico precoce possibilitaria intervenções que modificariam o curso da doença e assim, reduzir os agravos futuros à saúde da paciente.

Palavras-chave: Síndrome de Usher subtipo 2, Diagnóstico, Surdez congênita, Visão subnormal.

\section{ABSTRACT}

Objective: To describe the late diagnosis of a case of Usher Syndrome (US), analyze the clinical manifestations and classify the disease using ophthalmological findings, in addition to comparing them to the current literature. Case details: A 32-year-old woman, came to the consultation complaining of low visual acuity with worsening of night vision. She had lupus, systemic arterial hypertension, dyslipidemia, sensorineural hearing loss using a device, previous eye surgeries, phaco+lio in 2009 and 2016. Visual Acuity (VA) 20/50 in the Right Eye (OD) and 20/60 in the Left Eye (OS) with corrective lenses, tonometry $10 \mathrm{mmhg}$ $\mathrm{OD}$ and $13 \mathrm{mmhg} \mathrm{OS}$, biomicroscopy unchanged in both eyes (OU). On campimetry she presented peripheral Visual Field (VF) loss in OU. Funduscopy showed pale optic discs, vascular narrowing and bone spikes in OU. Final considerations: SU is characterized by the association of Retinosis Pigmentosa (RP) and congenital deafness, partial or total. It is an autosomal disease, inherited recessively. The clinical findings, together with the complementary exams, supported the diagnosis of RP and SU subtype 2. Early diagnosis would allow interventions that would modify the course of the disease and thus reduce future harm to the patient's health.

Key words: Usher Syndrome Subtype 2, Diagnosis, Congenital deafness, Low vision.

\footnotetext{
${ }^{1}$ Núcleo Médico de Oftalmologistas Ltda, Belo Horizonte - MG. *E-mail: viniciotadeu@yahoo.com.br
} 


\section{RESUMEN}

Objetivo: Describir el diagnóstico tardío de un caso de Síndrome de Usher (SU.), analizar las manifestaciones clínicas y clasificar la enfermedad mediante hallazgos of talmológicos, además de compararlos con la literatura actual. Detalles del caso: Una mujer de 32 años, acudió a la consulta quejándose de agudeza visual (AV) baja con empeoramiento de la visión nocturna. Tenía LUPUS, dislipidemia por hipertensión arterial sistémica, hipoacusia neurosensorial mediante dispositivo, cirugías oculares previas, faco + lio en 2009 y 2016 . En el examen AV 20/50 en el ojo derecho (OD) y 20/60 en el ojo izquierdo (OI) con lentes correctoras, tonometría de $10 \mathrm{mmhg}$ de OD y $13 \mathrm{mmhg}$ de Ol, biomicroscopía sin cambios en ambos ojos (AO). En la campimetría presentó pérdida de Campo Visual (CV) periférico en AO. La funduscopia mostró discos ópticos pálidos, estrechamiento vascular y picos óseos en la AO. Consideraciones finales: La SU se caracteriza por la asociación de Retinosis Pigmentosa (RP) y sordera congénita, parcial o total. Es una enfermedad autosómica, heredada de forma recesiva. Los hallazgos clínicos, junto con los exámenes complementarios, respaldaron el diagnóstico de PR y SU subtipo 2. El diagnóstico precoz permitiría intervenciones que modificarían el curso de la enfermedad y así reducir los daños futuros a la salud del paciente.

Palabras clave: Síndrome de Usher subtipo 2, Diagnóstico, Sordera congénita, Visión baja.

\section{INTRODUÇÃO}

A Síndrome de Usher (SU) foi descrita preliminarmente por Albert Von Graefe e Richard Liebreich médicos oftalmologistas de nacionalidade alemã, mas foi Charles Howard Usher, oftalmologista escocês, quem descobriu, em 1914, essa síndrome de herança genética, que passou então a ter seu nome (VON GRAFFE A, 1858; USHER C, 1914). A SU é uma doença autossômica de herança recessiva e se caracteriza pela associação de Retinite Pigmentar (RP) e hipoacusia neurossensorial congênita em graus variados (BONNET C e EL-AMRAOUI A, 2012).

A incidência estimada, entre alguns autores é de 3 a 4 em cada 100 mil pessoas na população mundial. Já na população de surdos prevalece na proporção de 3 a $6 \%$ e $18 \%$ dos casos de RP (MARAZITA ML, et al., 1993). A SU é clínica e geneticamente heterogênea, sendo dividida em três subtipos clínicos distintos, associados a vários loci genéticos. Os genes Usher codificam uma variedade de proteínas que são expressas no ouvido interno e na retina, onde desempenham funções essenciais no desenvolvimento e função das células ciliadas sensoriais e na manutenção dos fotorreceptores (TOMS M, et al., 2020)

A síndrome de Usher tipo 1 (Usher 1) é o subtipo mais grave em que os pacientes apresentam perda auditiva neurossensorial congênita bilateral severa a profunda, na maioria das vezes não progressiva, com arreflexia vestibular e cegueira noturna na infância. Usher tipo 1, representa aproximadamente $25-44 \%$ de todos os casos da síndrome (REINERS J, et al., 2006).

A síndrome de Usher tipo 2 (Usher 2) é o subtipo mais comum da doença, representando mais da $50 \%$ de todos os casos. A perda auditiva neurossensorial é normalmente descrita como, leve a moderada nas frequências baixa e severa a profunda nas frequências altas, disfunção vestibular e cegueira noturna (nictalopia) em adultos jovens (REINERS J, et al., 2006).

A perda auditiva é congênita e os bebês são avaliados por meio da triagem auditiva do recém-nascido, porém, se o exame não estiver disponível, o diag nóstico pode ser negligenciado até o final da primeira década de vida (BLANCO-KELLY F, et al., 2015). Embora considerada não progressiva, há evidências que indicam a progressão da perda auditiva ao longo dos anos, principalmente em Usher tipo 2A (REISSER CF, et al., 2002; SADEGHI M, et al., 2004).

As crianças se beneficiam com aparelhos auditivos convencionais e geralmente têm aquisição de fala próxima ao normal. Porém, com a progressão da perda auditiva, o implante coclear é indicado. Até $10 \%$ dos pacientes Usher tipo 2A faz uso de implante coclear (idade média: 59 anos), o que aumentou a inteligibilidade da fala, a qualidade de vida e a comunicação (HARTEL BP, et al., 2017).

A função vestibular está intacta em pacientes com Usher 2 e refletida nos marcos motores normais. No entanto, um estudo encontrou anormalidades vestibulares em quatro de cinco pacientes Usher 2 
geneticamente confirmados (MAGLIULO G, et al., 2017). Estudos relatam que episódios de vertigem foram queixados por vários pacientes, embora o equilíbrio clínico fosse normal, assim, sugeriram que alterações subclínicas no sistema vestibular deveriam ser mais pesquisadas (TOMS M, et al., 2020).

A síndrome de Usher tipo 3 (Usher 3) é o subtipo mais raro na maioria das populações, sendo responsável por aproximadamente 2 a $4 \%$ de todos os casos. A perda auditiva é de início pós-lingual e geralmente detectada na primeira década de vida, embora o início possa ser retardado até a vida adulta. É tipicamente de natureza progressiva sem problemas de equilíbrio, porém, com alterações visuais na vida adulta (MARAZITA ML, et al., 1993). Os aparelhos auditivos são benéficos no início do curso da doença, mas o implante coclear pode ser necessário com perda auditiva progressiva (PIETOLA L, et al., 2012).

A RP se desenvolver em todos os três subtipos de Usher, mas com início variável. Usher 1 é mais comumente pré-adolescente, com Usher 2 nas primeiras duas décadas de vida e os pacientes de Usher 3 tipicamente pós-púberes (MILLAN JM, et al., 2011). Normalmente, o primeiro sintoma manifestado é a cegueira noturna com perda progressiva do campo visual começando na periferia média, causada pela degeneração dos bastonetes. Eventualmente, progride para envolver os cones, resultando em perda de visão central e colorida (DAD S, et al., 2016).

Ao exame de fundo de olho desses pacientes é possível verif icar sinais característicos de RP, assim como, nervo óptico pálido, esclerose das arteríolas e dispersão de corantes no interior da retina no formato de espículas ósseas (FRIEDMAN TB, et al., 2011). Podendo também apresentar catarata capsular, nervo óptico com drusas, lesões atrófica na fóvea e edema macular. Também podem estar presentes: catarata subcapsular, drusa de nervo óptico, lesões foveais atróficas e edema macular cistóide (um inchaço da região central da retina), sendo este inchaço, melhor revelado pelos exames de imagem como angiografia de florescência ou pela Tomografia de Coerência Óptica (OCT) (WALIA S, et al., 2009).

Dentre os exames complementares a fundoscopia e de fundamental relevância, contudo, na ausência de achados na oftalmoscopia, sugerem-se testes de Campo Visual (CV) e Eletrorretinografia (ERG). Os exames de CV mostram graus variáveis de perda de sensibilidade em diferentes estágios da doença, porém não é específico (FRIEDMAN TB, et al., 2011).

A conf irmação final da degeneração retiniana é feita com ERG, que para pacientes com Usher apresentará diminuição da amplitude e retardo no tempo implícito de respostas dos cones e bastonetes. O ERG é o teste mais sensível para a detecção da degeneração retiniana e deve sempre ser feito na ausência dos achados of talmoscópicos clássicos, se houver forte suspeita de Usher. Além disso, pode revelar alterações retinianas que antecedem as alterações fundoscópicas, sendo o exame de escolha para o diagnóstico na infância (MENDIETA L, et al., 2005).

O objetivo deste estudo foi comunicar um caso de síndrome de Usher subtipo 2, analisar as manifestações clínicas e classificar a doença utilizando os achados oftalmológicos além de compará-los à literatura atual, bem como, evidenciar a importância do seu diagnóstico na prática clínica oftalmológica diária.

\section{DETALHAMENTO DO CASO}

Paciente de 32 anos, sexo feminino, procedente de lpatinga (MG), procurou atendimento of talmológico em nossos serviços queixando-se de Baixa Acuidade Visual (BAV) com piora da visão noturna. Como comorbidades, apresentava lúpus (em uso de Predinisona e Azatioprina), hipertensão arterial sistêmica, dislipidemia, hipoacusia neurossensorial em uso de aparelho desde a infância, duas cirurgias oculares prévias, Faco+LIO em 2009 e 2016. Nega história de consanguinidade entre pais. Afirma ter atendimentos of talmológicosprévios, sem diagnóstico para sua condição. Ao exame apresentava Acuidade Visual AV 20/50 no Olho Direito (OD) e 20/60 Olho Esquerdo (OE) com lentes corretivas, tonometria $10 \mathrm{mmhg}(\mathrm{OD})$ e $13 \mathrm{mmhg}$ $(\mathrm{OE})$, biomicroscopia sem alteração em $\mathrm{AO}$. À campimetria evidenciou perda de campo visual periférico em $A O$, o que determina a nictalopia (Figura 1). 
Figura 1 - Campimetria da paciente com SU revelando avançado def eito de campo visual, com campo tubular em AO. Devido à diminuição da função dos bastonetes que estão localizados na periferia da retina, o que causa queixa de nictalopia.

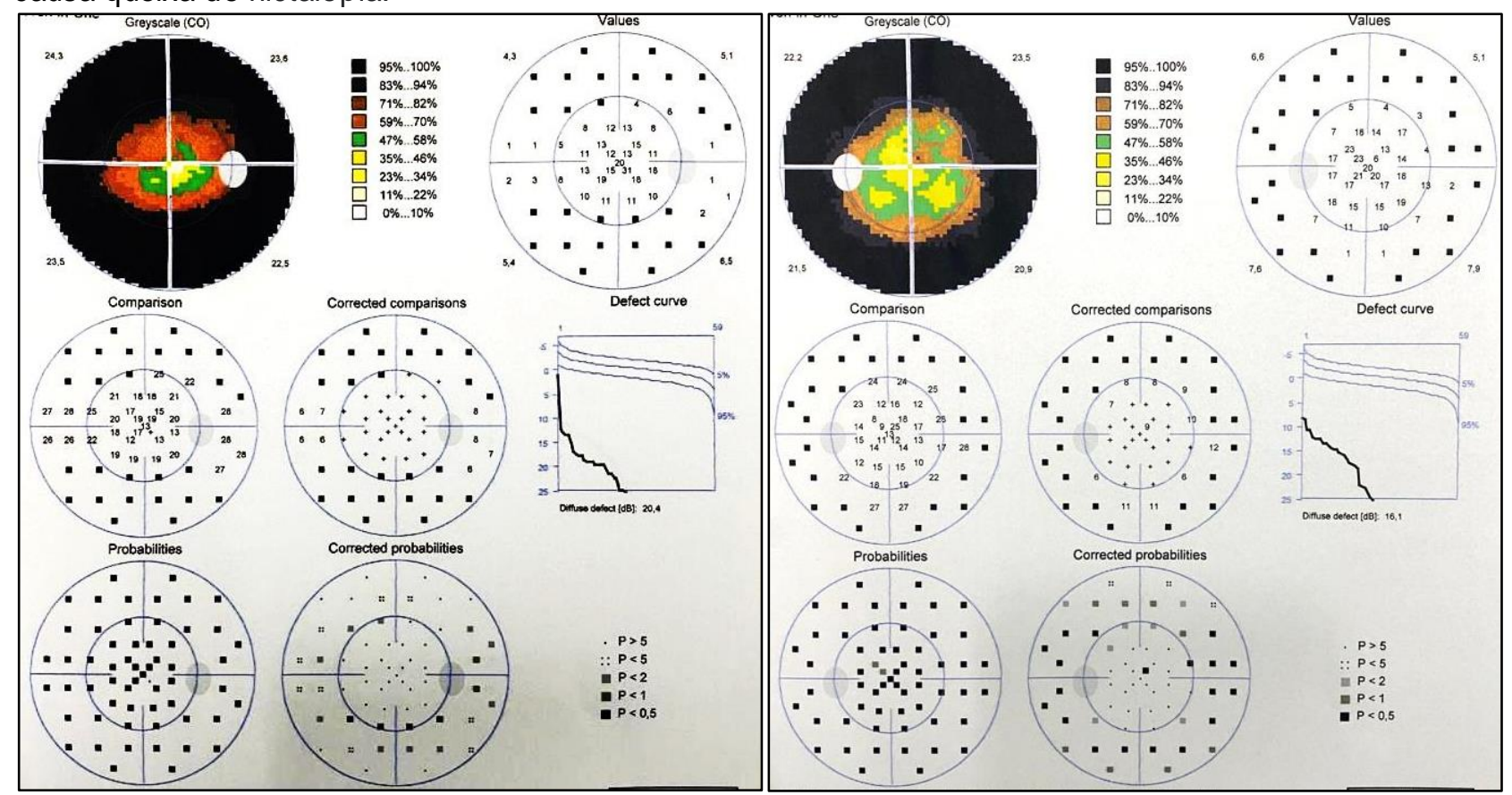

Fonte: Coelho VTS, et al., 2021.

À fundoscopia foram evidenciados discos ópticos pálidos, estreitamento vascular e espículas ósseas AO (Figura 2). Com a história do caso e com os achados do exame físico, em conjunto com os exames complementares, confirmaram para o diagnóstico de RP e SU subtipo 2.

Figura 2. Retinografia colorida apresentando os achados clínicos fundoscópicos típicos de retinose pigmentar. Fundo de olho mostrando discos pálidos, atenuação vascular, intensa atrofia retiniana difusa com ilha preservada na mácula e espículas ósseas em AO.
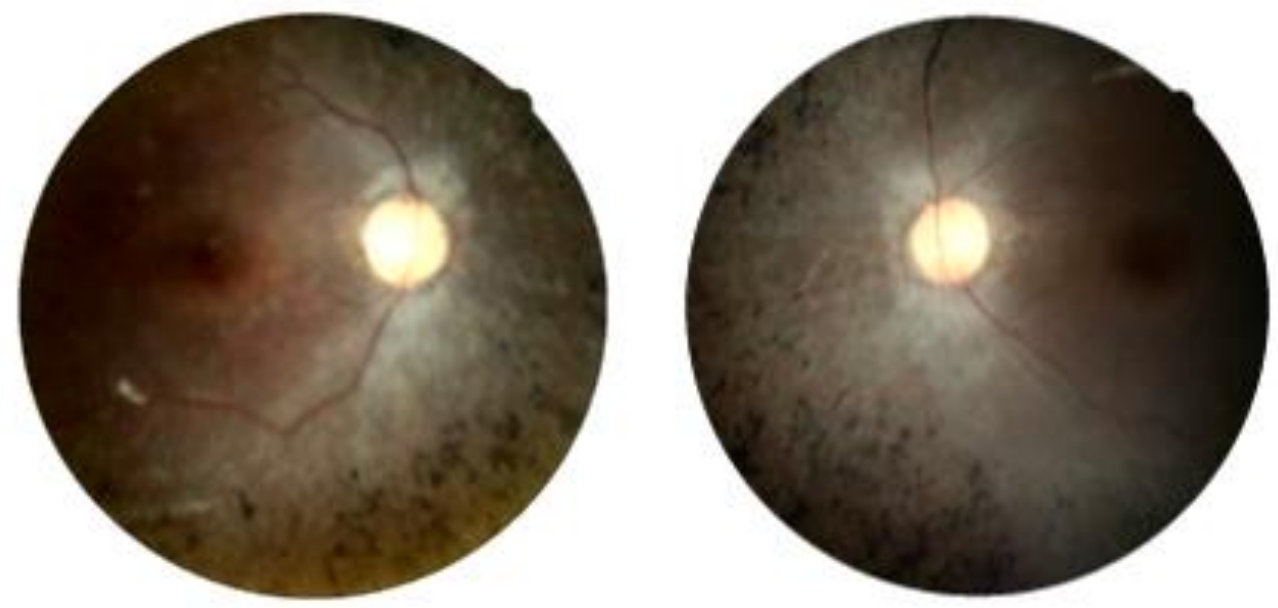

Fonte: Coelho VTS, et al., 2021.

\section{DISCUSSÃO}

A SU se caracterizada por apresentar duplo comprometimento sensorial, dos sistemas auditivos associados à diminuição progressiva da visão, apresentando como sintomas basais a nictalopia e diminuição 
de campo visual periférico (BONNET C e EL-AMRAOUI A, 2012; MATHEUS RJ, et al., 2021). Ao analisar o caso, pode-se inferir que a paciente apresenta SU tipo 2 , devido às alterações visuais congênita associada à diminuição da acuidade visual na vida adulta e ao avanço da retinose pigmentar.

O diagnóstico correto e precoce é importante, pois ações para melhorar a condição de vida e o aconselhamento genético devem ser instituídas o mais breve possível (MENDIETA L, et al., 2005). Diagnosticar a síndrome somente pela semiologia clinica pode ser desafiador, sendo necessários exames complementares, como retinografia, campimetria e em casos mais complexos podem exigir exames mais elaborados como, ERG, OCT e Retinografia com autofluorescência ou até mesmo análise molecular, apresentando atualmente onze genes associados à doença (JOURET G, et al., 2019).

Usher 2 deve ser considerado em pacientes com características típicas de perda auditiva. O diagnóstico de Usher 2 ou 3 é feito após a detecção de sintomas ou sinais visuais por meio de exames de rotina ou ERG. Um estudo de metanálise com dados de sequenciamento gênico realizado nos Estados Unidos indica que $7,5 \%$ dos pacientes com "surdez isolada" têm mutações nos genes Usher e podem ter alto risco de desenvolver RP. De tal modo que, com a disponibilidade dos genes Usher em banco de dados auxilia no diagnóstico genético precoce (JOURET G, et al., 2019).

O exame de campimetria visual, mesmo não sendo específico, auxiliou no diagnóstico, evidenciando profunda perda do campo visual periférica, no caso em estudo (Figura 1). Fishman GA, et al. (1983), relatam que a gravidade da audição diminuída, não se vincula com a gravidade da ausência da $A V$ central, mas com a influência da perda do CV periférico. Sendo assim, com a progressão da perda do CV periférico da paciente, consequentemente ocorrerá uma piora da capacidade auditiva, sendo esta já muito comprometida.

A retinografia colorida é um exame que consiste em visualizar e registrar o fundo de olho. Este exame permitiu evidenciar discos ópticos pálidos, halo retiniano preservado na mácula, estreitamento vas cular e espículas ósseas espalhadas na periferia da retina e despigmentação com atrofia do Epitélio Pigmentar da Retina em AO (Figura 2). Estes achados assemelham com as características descritas na literatura, imagem da retina de um paciente com SU tipo 2 com variantes heterozigotas compostas no gene USH2A, corroborando para um diagnóstico mais preciso do caso em estudo. Três genes subjacentes ao Usher 2 foram identificados como USH2A (USH2A), ADGRV1 (USH2C) e WHRN (USH2D) (TOMS M, et al., 2020). As mutações USH2A são as causas mais comuns da SU, sendo responsáveis por cerca de $80 \%$ dos casos de Usher 2 (LE QUESNE STABEJ P, et al., 2012).

O manejo da Síndrome é evitar o avanço da RP permitindo melhor condição de vida ao paciente. Atualmente, existem vários estudos em andamento na literatura sobre novas estratégias terapêuticas, como implantes retinais artificiais, terapia farmacológica e terapia gênica (DJILAS M, et al., 2011; GENEAD MA e FISHMAN GA, 2010; ZOU J, et al., 2011).

Os implantes artificiais retinianos envolvem o contato de microchips com a camada fotorreceptora da retina ou implantes de eletrodos no interior da retina junto às células ganglionares (ZRENNER E, et al., 2011). Existem estudos em andamento avaliando a possibilidade de transplante de epitélio pigmentar da retina, fotorreceptores ou células-tronco, com resultados promissores como aumento da acuidade visual e proteção dos neurônios da retina (SEILER MJ e ARAMANT RB, 2005; MEYER JS, et al., 2006).

O olho é um órgão atraente para aplicações de modelos terapêuticas devido à sua acessibilidade $\mathrm{e}$ privilégio imunológico. Embora atualmente não exista cura disponível, existem diversas estratégias terapêuticas em desenvolvimento para RP relacionada à Usher e outras doenças retinianas hereditárias. Estas estratégias incluem a substituição de genes, edição de genes, supressão antisense e abordagens baseadas em oligonucleotídeos antisense. A maioria destes estudos foi realizada utilizando fibroblastos originários de pacientes acometidos pela sínd rome (TOMS M, et al., 2020).

Em relação à abordagem farmacológica, há evidências de que a suplementação de vitamina $A$ resulta em perda mais lenta do campo visual, no entanto, o monitoramento periódico deve ser mantido para avaliar possíveis ef eitos colaterais, como osteoporose. O ácido docosahexaenóico, um ácido graxo ômega-3, tem sido utilizado para tratamento porque as rodopsinas e as iodopsinas contêm altos níveis dessa substância e os pacientes com RP têm níveis séricos mais baixos (HARTONG DT, et al., 2006). 
Para o tratamento do edema macular cistóide as injeções intravítreas de corticosteroides ou fatores de crescimento endotelial anti-vascular (anti-VEGF), como o bevacizumabe, podem ser indicadas, além de inibidores da anidrase carbônica orais ou tópicos, que podem fornecer uma melhora transitória na acuidade visual nesses pacientes (BUCHAIM G, et al., 2013).

A SU é um distúrbio com vasta heterogeneidade clínica e genética, normalmente resultando em perda sensorial dupla significativa, causando grande impacto na qualidade de vida do paciente. Diversos sinais, sintomas e síndromes podem correlacionar com a RP, sendo a SU a mais comumente correlacionada. Assim, é necessário que os oftalmologistas tenham noção destas, não apenas para um diagnóstico apropriado, mas também orientar de maneira adequada os seus pacientes (TOMS M, et al., 2020).

Neste caso, foi possível observar o padrão clínico característico da SU subtipo 2, pois a paciente apresentou sintomas iniciais de RP em idade adulta associado à surdez congênita, o que corroboram para a melhor caracterização e diagnóstico da doença. Uma análise suplementar é indispensável para definir melhores correlações genótipo-fenótipo para cada subtipo clínico, para antecipar o prognóstico e assim delinear de forma mais assertiva, o aconselhamento genético da paciente.

\section{REFERÊNCIAS}

1. BLANCO-KELLYF, et al. Clinical aspects of Usher syndrome and the USH2A gene in a cohort of 433 patients. Joumal American Medical Association Ophthalmology, 2015;133:157-164.

2. BONNET C, EI-AMRAOUI A. Usher syndrome (sensorineural deafness and retinitis pigmentosa): pathogenesis, molecular diagnosis and therapeutic approaches. Curr Opin Neurol., 2012;25(1): 42-9.

3. BUCHAIM G, et al. Implante intravítreo de liberação crônica de dexametasona (Ozurdex $®$ ) para o tratamento de edema macular por retinose pigmentar: relato de caso. Arq Bras Oftalmol., 2013;76(6): 377-9.

4. DAD S, et al. Usher syndrome in Denmark: mutation spectrum and some clinical observations. Mol Genet Genomic Med, 2016; 4: 527-539.

5. DJILAS M, et al. Three-dimensional electrode arrays for retinal prostheses: modeling, geometry optimization and experimental validation. J Neural Eng., 2011;8(4).

6. FISHMAN GA, et al. Usher's syndrome. Ophthalmic and neuro-otologic findings suggesting genetic heterogeneity. Arch Ophthalmol, 1983;101:1367-74.

7. FRIEDMAN TB, et al. Usher Syndrome: Hearing Loss with Vision Loss. Adv Otorhinolaryngol, 2011;70:56-65.

8. GENEAD MA, FISHMAN GA. Efficacy of sustained topical dorzolamide therapy for cystic macular lesions in patients with retinitis pigmentosa and Usher syndrome. Arch Ophthalmol., 2010;128(9):1146-50.

9. HARTEL BP, et al. Cochlear implantation in patients with Usher syndrome type lla increases performance and quality of life. Otol Neurotol, 2017;38: e120-e127.

10. HARTONG DT, et al. Retinitis Pigmentosa. Lancet. 2006; 368(9549): 1795-809.

11. JOURET G, et al. Genetics os Usher Syndrome: New Insights From a Metaanalysis. Otol Neurotol., 2019; 40(1):1219.

12. LE QUESNE STABEJ $P$, et al. Comprehensive sequence analysis of nine Usher syndrome genes in the UK National Collaborative Usher Study. J Med Genet, 2012;49:27-36.

13. MAGLIULO G, et al. Usher's syndrome type II: a comparative study of genetic mutations and vestibular system evaluation. Otolaryngol Head Neck Surg, 2017; 157: 853-860.

14. MARAZITA ML, et al. Genetic epidemiologic studies of early-onset deafness in the United States school-age population. Am J Med Genet, 1993; 46: 486-491.

15. MATHEUS RJ, et al. Ocular manifestations and conduct in Usher Syndrome. eOftalmo., 2021;7(1):25-30.

16. MENDIETA $L$, et al. Acuidade visual e eletrorretinografia de campo total em pacientes com síndrome de Usher. Arq Bras Oftalmol., 2005;68(2):171-6.

17. MEYER JS, et al. Embryonic stem cell-derived neural progenitors incorporate into degenerating retina and enhance survival of host photoreceptors. Stem Cells., 2006; 24(2):274-83.

18. MILLAN JM, et al. An update on the genetics of Usher syndrome. J Ophthalmol, 2011;417217.

19. PIETOLA L, et al. Speech recognition and communication outcomes with cochlear implantation in Usher syndrome type 3. Otol Neurotol, 2012;33: 38-41.

20. REINERS J, et al. Molecular basis of human Usher syndrome: deciphering the meshes of the Usher protein network provides insights into the pathomechanisms of the Usher disease. Exp Eye Res, 2006;83: 97-119. 
21. REISSER CF, et al. Hearing loss in Usher syndrome type II is nonprogressive. Ann Otol Rhinol Laryngol, 2002; 111: $1108-1111$.

22. SADEGHI M, et al. Audiological findings in Usher syndrome types lla and II (non-Ila). Int J Audiol, 2004;43: 136-143.

23. SEILER MJ, ARAMANT RB. Transplantation of neuroblastic progenitor cells as a sheet preserves and restores retinal function. Semin Ophthalmol., 2005;20(1): 31-42.

24. TOMS M, et al. Usher syndrome: clinical features, molecular genetics and advancing therapeutics. Ther Adv Ophthalmol, 2020;12:25-52.

25. USHER C. On the inheritance of retinis pigmentosa, with notes of cases. Royal London Ophta Imol Hosp Rep., 1914; 19: 130-236.

26. VON GRAFFE A. Vereizelle Beobachtungen und bemerkungen-Exceptionnelles verhaller des Gesichts feldes bei Pigmentenarter der Netzhalt. Arch Klin Ophtalmol. 1858; 4:250-3.

27. WALIA S, et al: Prevalence of cystic macular lesions in patients with Usher II syndrome. Eye (Lond), 2009;23: 12061209.

28. ZOU J, et al. Whirlin replacement restores the formation of the USH2 protein complex in whirlin knockout photoreceptors. Invest Ophthalmol Vis Sci., 2011;52(5): 2343-51.

29. ZRENNER E, et al. Subretinal electronic chips allow blind patients to read letters and combine them to words. Proc Biol Sci., 2011;278(1711):1489-97. 